\title{
ON THE SHORTEST PATH THROUGH A NUMBER OF POINTS
}

\section{S. VERBLUNSKY}

1. Introduction. Let $O$ denote a bounded open set in the $x, y$ plane, with boundary of zero measure. There is a least absolute positive constant $C$ with the following property: given $\epsilon>0$, there is an $N=N(O, \epsilon)$ such that, if $n>N$, then any $n$ points in $O$ are on ath of length less than $(C+\epsilon)(n \cdot m O)^{1 / 2}$. Here, $m O$ is the measure of $O$. The path is not necessarily contained in $O$ if $O$ is not convex.

A set $O$ of the kind specified is contained in a finite number of squares of total area arbitrarily near to $m O$. The above assertion is easily seen to be a consequence of the following. Given $\epsilon>0$, there is an $N(\epsilon)$ such that, if $n>N(\epsilon)$, then any $n$ points in a unit square are on a path of length less than $(C+\epsilon) n^{1 / 2}$.

A simple argument (see $\S 2$ ) will show that $C \leqq 2$. On the other hand, it has been pointed out by Fejes (Math. Zeit. vol. 46 (1940) p. 85) that $C \geqq C_{0}$ where $C_{0}=2^{1 / 2} 3^{-1 / 4}$. For we can arrange a large number $n$ of points in a unit square so as to form a lattice of equilateral triangles, and a shortest path through the points is then seen to be asymptotically equal to $C_{0} n^{1 / 2}$. That $C \leqq(2.8)^{1 / 2}$ is a consequence of the following theorem.

Theorem I. Given $n$ points in a unit square, there is a path through the points of length less than $2+(2.8 n)^{1 / 2}$.

This theorem can be deduced from the following theorem.

Theorem II. Given $\nu$ points $P_{i}\left(x_{i} y_{i}\right)$ in the strip $0 \leqq y \leqq 1$ with $x_{1}$ $\leqq x_{2} \leqq \cdots \leqq x_{\nu}$, there is a path whose first point is $P_{1}$, whose last point is $P_{j}$ say, which contains the $\nu$ points, and whose length does not exceed $x_{j}-x_{1}+.7 \nu$.

By examining the case in which the points $P_{i}$ are $(0,0),\left(3^{-1 / 2}, 1\right)$, $\left(2 \cdot 3^{-1 / 2}, 0\right), \cdots$, we see that, in Theorem II, .7 cannot be replaced by a number less than $3^{-1 / 2}$.

2. Proof that $C \leqq 2$. The simple argument which implies that $C \leqq 2$ is as follows. Divide the square into $m$ strips of breadth $m^{-1}$. Let $A_{i} B_{i}(i=1, \cdots, m)$ denote the longitudinal central lines of these strips. Then the path

$$
p ; \quad A_{1} B_{1} B_{2} A_{2} A_{3} \cdots
$$
1950.

Presented to the Society, December 29, 1950; received by the editors October 31, 
is of length $m+1-m^{-1}$. Each of the $n$ points is at a distance not greater than $m^{-1 / 2}$ from $p$. We connect it to $p$ by a segment of length not greater than $m^{-1} / 2$. Then, if we count each segment twice, we obtain a path which contains the $n$ points and is of length not exceeding $m+1+(n-1) m^{-1}$. It suffices to choose $m=1+\left[n^{1 / 2}\right]$, where $[x]$ denotes the greatest integer less than $x$.

3. Theorem II implies Theorem I. Turning now to the deduction of Theorem I from Theorem II, we suppose that the square has its sides parallel to the coordinate axes. Divide the square into $m$ strips of breadth $m^{-1}$, parallel to the $x$ axis. Consider any one of these strips which contains at least one, say $\nu$, of the $n$ points. By Theorem II (with a change of scale), there is a path containing the $\nu$ points of length not exceeding

$$
d+.7 v m^{-1}, \quad d=\xi_{l}-\xi_{f} \geqq 0,
$$

where $\xi_{f}, \xi_{l}$ are the abcissae of the first and last points on the path. If $\nu=1$, we can put $\xi_{f}=\xi_{l}$.

The number of such strips is, say, $\mu \leqq m$. We enumerate these strips in order of increasing $y$. Call the path constructed in strip $i$ the path $i$. Connect the last point of path 1 with the last point of path 2 by a segment; connect the first point of path 2 with the first point of path 3 by a segment; and so on. We thus obtain a path which contains the $n$ points, and is of length not exceeding

$$
\sum d_{i}+.7 m^{-1} \sum \nu_{i}+s
$$

where $s$ is the sum of the $\mu-1$ segments which have been introduced.

The length of each segment does not exceed the sum of its projections on the coordinate axes. If we project the $\mu-1$ segments on the $y$ axis, the amount of overlapping does not exceed $(\mu-2) m^{-1}$. Hence the sum of the projections on the $y$ axis does not exceed

$$
1+(\mu-2) m^{-1} \leqq 2-2 m^{-1} .
$$

The projection of the first segment on the $x$ axis can be added to $d_{1}$ if $\xi_{l_{2}}>\xi_{l_{1}}$ to produce a modified term which does not exceed $1-\xi_{f_{1}}$; it can be added to $d_{2}$ if $\xi_{l_{2}}<\xi_{l_{1}}$ to produce a modified term which does not exceed $1-\xi_{f_{2}}$. The projection of the second segment on the $x$ axis can be added to $d_{2}$ (or to the modified $d_{2}$ ) if $\xi_{f_{3}}<\xi_{f_{2}}$ to produce a term which does not exceed 1 ; it can be added to $d_{3}$ if $\xi_{f_{3}}>\xi_{f_{2}}$ to produce a modified term which does not exceed $\xi_{l_{3}}$; and so on. Hence the length of the path does not exceed

$$
m+2-2 m^{-1}+.7 n m^{-1} \text {. }
$$


Choose $m=1+\left[(.7 n)^{1 / 2}\right]$, and the theorem follows.

4. Reduction of proof of Theorem II to the lemma. Let $P_{2}^{\prime}, \cdots, P_{v}^{\prime}$ denote a permutation of $P_{2}, \cdots, P_{v}$. The only paths we need consider are those consisting of $\nu-1$ segments

$$
P_{1} P_{2}^{\prime}, P_{2}^{\prime} P_{3}^{\prime}, \cdots, P_{v-1}^{\prime} P_{v}^{\prime}
$$

Such paths will be called admissible paths through $P_{1}, P_{2}, \cdots, P_{v}$. We denote the path (1), as well as its length, by the symbol $P_{1} P_{2}^{\prime} \cdots P_{\prime}^{\prime}$. The assertion of Theorem II is that there is an admissible path $P_{1} \cdots P_{j}$ through $P_{1}, P_{2}, \cdots, P_{v}$ of length not exceeding $x_{j}-x_{1}+.7 \nu$.

On grounds of continuity, we may suppose that $x_{1}<x_{2}<\cdots<x_{\text {n }}$, provided that we prove

$$
P_{1} \cdots P_{j}<x_{j}-x_{1}+.7 \nu .
$$

Call this result Theorem $\mathrm{II}^{\prime}$. It is a consequence of the following lemma.

Lemma. Given $\nu$ points $P_{i}\left(x_{i}, y_{i}\right)$ with

$$
x_{1}<x_{2}<\cdots<x_{\nu}, \quad 0 \leqq y_{i} \leqq 1,
$$

no two of which are at a distance less than .35 , there is an admissible path $P_{1} \cdots P_{j}$ through $P_{1}, P_{2}, \cdots, P_{\nu}$ of length less than $x_{j}-x_{1}+.7 \nu$.

For, assuming the lemma, we need only consider the case where at least one distance is less than .35, and this case of Theorem $\mathrm{II}^{\prime}$ can be proved by induction. It is true for $\nu=2$. Suppose then that $\nu>2$. By hypothesis, there is a pair of points at a distance less than .35, say $P, Q$ with $x_{P}<x_{Q}$. Then $Q \neq P_{1}$. Consider the $\nu-1$ points other than $Q$. By the inductive hypothesis, there is an admissible path $P_{1} \ldots P_{j}$ through them such that

$$
P_{1} \cdots P_{j}<x_{j}-x_{1}+.7(\nu-1) \text {. }
$$

If $P_{j}=P$, then

$$
\begin{aligned}
P_{1} \cdots P_{j} Q & <x_{j}-x_{1}+.7(\nu-1)+.35 \\
& <x_{Q}-x_{1}+.7 \nu,
\end{aligned}
$$

and $P_{1} \cdots P_{j} Q$ is a path as required. If $P_{j} \neq P$, consider the symbol obtained from $P_{1} \cdots P_{j}$ by inserting $Q$ immediately after $P$. The corresponding path is of length less than

$$
P_{1} \cdots P_{j}+P Q+Q P<x_{j}-x_{1}+.7 \nu,
$$


and is a path as required.

5. Proof of the lemma. The idea of the proof of the lemma is as follows. We attempt to construct successive portions of an admissible path. If

$$
P_{1} P_{2}<x_{2}-x_{1}+.7 \text {, }
$$

then $P_{1} P_{2}$ is the first segment of the path. If (2) is not satisfied, and if $\nu>2$, we consider the paths $P_{1} P_{2} P_{3}, P_{1} P_{3} P_{2}$. If one of the inequalities

$$
P_{1} P_{2} P_{3}<x_{3}-x_{1}+1.4, \quad P_{1} P_{3} P_{2}<x_{2}-x_{1}+1.4
$$

is satisfied, we choose such a one, and the corresponding pair of segments is the first pair of the path. If none of the inequalities (2), (3) are satisfied, and if $\nu>3$, we consider the four paths $P_{1} P_{2} P_{3} P_{4}$, $P_{1} P_{2} P_{4} P_{3}, P_{1} P_{3} P_{2} P_{4}, P_{1} P_{3} P_{4} P_{2}$. If one of them, say $P_{1} \cdots P_{r}$, satisfies

$$
P_{1} \cdots P_{r}<x_{r}-x_{1}+2.1,
$$

we choose such a one, and the corresponding triad of segments is the first triad of the path. If none of the inequalities (2), (3), or (4) are satisfied, and if $\nu>4$, we consider the four paths $P_{1} P_{2} P_{3} P_{4} P_{5}$, $P_{1} P_{2} P_{4} P_{3} P_{5}, P_{1} P_{2} P_{3} P_{5} P_{4}, P_{1} P_{2} P_{4} P_{6} P_{3}$. It turns out that at least one of these, say $P_{1} \cdots P_{r}$, satisfies

$$
P_{1} \cdots P_{r}<x_{r}-x_{1}+2.8 \text {. }
$$

We choose such a one, and the corresponding quadruple of segments is the first quadruple of the path. When none of the inequalities (2), (3), or (4) are satisfied, we have

$$
P_{1} P_{2} P_{3} P_{4}<x_{4}-x_{1}+2.8 \text {. }
$$

For if

$$
P_{1} P_{2} P_{3} P_{4} \geqq x_{4}-x_{1}+2.8,
$$

then since

$$
P_{i} P_{i+1} \leqq x_{i+1}-x_{i}+\left|y_{i+1}-y_{i}\right| \quad(i=1,2,3)
$$

we have

$$
\sum_{i=1}^{3}\left|y_{i+1}-y_{i}\right| \geqq 2.8 .
$$

But each term does not exceed 1. Hence each term is at least .8. We 
may suppose, by a reflection in $y=1 / 2$ if necessary, that $y_{1}>y_{2}$. Then

$$
y_{1}>.8, \quad y_{2}<.2, \quad y_{3}>.8, \quad y_{4}<.2 \text {. }
$$

This is the case $\alpha \delta \alpha \delta$ in the notation of $\S 7$. It is shown in $\S 8$ that either $P_{1} P_{3} P_{2} P_{4}$ is an admissible path through $P_{1}, P_{2}, P_{3}, P_{4}$ which satisfies (4) (a possibility which is here excluded by hypothesis), or else

$$
P_{1} P_{2} P_{3}<x_{3}-x_{1}+1.5
$$

A fortiori

$$
P_{1} P_{2} P_{3} P_{4}<x_{4}-x_{1}+2.5,
$$

which contradicts (7).

6. Proof of lemma continued. The result so far is: if $\nu>4$, then we can construct a portion $P_{1} \cdots P_{r}(r>1)$ of the required path, consisting of $k$ segments, such that

$$
P_{1} \cdots P_{r}<x_{r}-x_{1}+.7 k .
$$

If $\nu=2,3,4$, then either the same conclusion holds, or else the construction fails.

When the construction does not fail, and the portion constructed does not contain all the $\nu$ points, we attempt to repeat the argument starting from $P_{r}$ and ignoring the points which precede it in the symbol $P_{1} \cdots P_{r}$. It is important to notice that with the above meaning of $k$, the letters in the symbol $P_{1} \cdots P_{r}$ are a permutation of $P_{1}, P_{2}, \cdots P_{k+1}$. Thus, if we ignore the points which precede $P_{r}$ in the symbol $P_{1} \cdots P_{r}$, we are left with $P_{r}$ and certain $P_{\rho}$ where $\rho>r$.

By the statements of $\S 5$, the construction can always be repeated if there are at least four points not on the portion already constructed. If there are only one, two, or three such points, the repetition may or may not be possible. We repeat the construction if possible. We then obtain a second portion $P_{r} \cdots P_{s}(s>r)$ of the required path, consisting of $\kappa$ segments, such that

$$
P_{r} \cdots P_{s}<x_{8}-x_{1}+.7 \kappa .
$$

If $P_{1} \cdots P_{r} \cdots P_{s}$ does not contain all the $\nu$ points, we attempt to repeat the argument starting from $P_{s}$ and ignoring the points which precede it in the symbol $P_{1} \cdots P_{r} \cdots P_{s}$; and so on. The process can come to an end only in one of the following four cases.

(i) All the $\nu$ points are on the path constructed. By adding the in- 
equalities $(8),(9), \cdots$, we find that the path $P_{1} \cdots P_{j}$, say, satisfies

$$
P_{1} \cdots P_{j}<x_{j}-x_{1}+.7(\nu-1) .
$$

(ii) One point is not on the path constructed. Then since, at any stage, the points not on the portion so far constructed have suffixes greater than those on the portions constructed, the point not on the path is $P_{\nu}$. On adding (8), (9), . . we obtain for the path $P_{1} \cdots P_{t}$ through the $\nu-1$ points $P_{1}, P_{2}, \cdots, P_{\nu-1}$,

$$
P_{1} \cdots P_{t}<x_{t}-x_{1}+.7(\nu-2) \text {. }
$$

But

$$
P_{t} P_{\nu}<x_{\nu}-x_{t}+1
$$

Hence

$$
P_{1} \cdots P_{t} P_{\nu}<x_{\nu}-x_{1}+.7 \nu .
$$

(iii) Two points are not on the path constructed. For the reason mentioned in (ii), the two points are $P_{v-1}, P_{v}$. The path constructed, $P_{1} \cdots P_{t}$, satisfies

$$
P_{1} \cdots P_{t}<x_{t}-x_{1}+.7(\nu-3) .
$$

But

$$
P_{t} P_{\nu-1}<x_{v-1}-x_{t}+1, \quad P_{\nu-1} P_{v}<x_{\nu}-x_{\nu-1}+1
$$

Hence

$$
P_{1} \cdots P_{t} P_{\nu-1} P_{\nu}<x_{v}-x_{1}+.7 \nu .
$$

(iv) Three points are not on the path constructed. For the reason mentioned in (ii), the three points are $P_{\nu-2}, P_{\nu-1}, P_{\nu}$. The path constructed, $P_{1} \cdots P_{t}$, satisfies

$$
P_{1} \cdots P_{t}<x_{t}-x_{1}+.7(\nu-4) \text {. }
$$

By $\$ 5$ (replacing $P_{1}, P_{2}, P_{3}, P_{4}$ by $P_{t}, P_{\nu-2}, P_{\nu-1}, P_{\nu}$ respectively), either there is a path through $P_{t}, P_{\nu-2}, P_{\nu-1}, P_{\nu}$, beginning at $P_{t}$, ending at $\boldsymbol{P}_{\boldsymbol{j}}$, and satisfying

$$
P_{t} \cdots P_{j}<x_{j}-x_{t}+2.1
$$

or else

$$
P_{t} P_{\nu-2} P_{\nu-1} P_{\nu}<x_{\nu}-x_{t}+2.8 \text {. }
$$

In the first case $P_{1} \cdots P_{t} \cdots P_{j}$ is a path as required; in the second 
case $P_{1} \cdots P_{t} P_{v-2} P_{v-1} P_{v}$ is such a path.

We thus see that if we justify the assertions of $\$ 5$, the lemma will follow.

7. Proof of lemma completed. Divide the strip $0 \leqq y \leqq 1$ into four strips

$\alpha: 1 \geqq y \geqq 4 / 5, \beta: 4 / 5 \geqq y \geqq 1 / 2, \gamma: 1 / 2 \geqq y \geqq 1 / 5, \delta: 1 / 5 \geqq y \geqq 0$.

The possible sets $P_{1}, P_{2}, \cdots, P_{\nu}$ can be divided into four classes (or cases) $\alpha, \beta, \gamma, \delta$ according as $P_{1}$ is in $\alpha, \beta, \gamma$, or $\delta$. Each class can be divided into four subclasses according as $P_{2}$ is in $\alpha, \beta, \gamma$, or $\delta$; and so on. Thus, the class $\alpha \delta \alpha \delta$ is the class in which $P_{1}$ is in $\alpha, P_{2}$ in $\delta$, $P_{3}$ in $\alpha$, and $P_{4}$ in $\delta$. There is some overlapping among the classes, but this is irrelevant.

We need only consider the cases $\alpha$ and $\beta$, since $\gamma$ and $\delta$ are obtained from them by a reflection in the line $y=1 / 2$. The case $\beta$ is easier than $\alpha$. In case $\alpha$ we consider the subcases $\alpha \alpha, \cdots, \alpha \delta$ and see if (2) is satisfied. For any subcase in which this is not so, we consider its subcases, and see if one of (3) is satisfied. For any subcase in which this is not so, we consider its subcases, and see if (4) is satisfied. The reader will have an adequate idea of the method, if we consider in detail the most difficult of the subcases, and the only one which requires the consideration of $P_{5}$, namely, $\alpha \delta \alpha \delta$.

We use the elementary fact that if $A B$ is a segment which makes an acute angle $\phi$ with a given line, then

$$
A B=A B \cos \phi+A B \sin \phi \tan \frac{\phi}{2} .
$$

In the applications, we know upper bounds $h, v$ for $A B \cos \phi, A B \sin \phi$ respectively, and an upper bound $\alpha<\pi / 2$ for $\phi$. We can then infer that

$$
A B \leqq h+v \tan \frac{\alpha}{2} .
$$

An example of this is when two of the points $P_{i}$, say $P_{1}, P_{2}$, both belong to $\alpha$ or to $\delta$. Since, by hypothesis,

$$
P_{1} P_{2}>.35, \quad\left|y_{1}-y_{2}\right| \leqq .2,
$$

we have $\sin \phi<4 / 7$, where $\phi$ is the inclination of $P_{1} P_{2}$ to the $x$-axis. Then $\tan (\phi / 2)<1 / 3$, and

$$
P_{1} P_{2} \leqq x_{2}-x_{1}+\left|y_{2}-y_{1}\right| / 3<x_{2}-x_{1}+.07 \text {. }
$$


The case $\alpha \delta \alpha \delta$. We use the abbreviations

$$
d_{2}=x_{3}-x_{2}, \quad d_{3}=x_{4}-x_{3}, \quad d_{4}=x_{5}-x_{4} .
$$

We have

$$
\begin{aligned}
P_{1} P_{3} P_{2} P_{4} & \leqq x_{3}-x_{1}+.07+\left(1+d_{2}^{2}\right)^{1 / 2}+x_{4}-x_{2}+.07 \\
& \leqq x_{4}-x_{1}+d_{2}+\left(1+d_{2}^{2}\right)^{1 / 2}+.14 .
\end{aligned}
$$

Hence

$$
P_{1} P_{3} P_{2} P_{4}<x_{4}-x_{1}+2.1
$$

if $d_{2} \leqq .72$. We may therefore suppose that $d_{2}>.72$. Then if $\epsilon$ is the inclination of $P_{1} P_{8}$ to the $x$-axis,

$$
\tan \epsilon<5 / 18, \quad \tan \frac{\epsilon}{2}<.14
$$

and so

$$
P_{1} P_{3} \leqq x_{8}-x_{1}+.14\left|y_{1}-y_{3}\right|<x_{8}-x_{1}+.03,
$$

and similarly,

$$
P_{2} P_{4}<x_{4}-x_{2}+.03 .
$$

We can therefore repeat the above argument with .03 in place of .07 . We then find that (10) holds if $d_{2} \leqq .77$. We may therefore suppose that $d_{2}>.77$. Then if $\theta$ is the inclination of $P_{2} P_{3}$ to the $x$-axis,

$$
\tan \theta<\frac{1}{.77}, \quad \tan \frac{\theta}{2}<.5, \quad P_{2} P_{3}<d_{2}+.5,
$$

and so

$$
P_{1} P_{2} P_{3}<x_{3}-x_{1}+1.5 \text {. }
$$

We now consider the four subcases.

Case $\alpha \delta \alpha \delta \alpha$. We have

$$
\begin{aligned}
P_{1} P_{2} P_{4} P_{3} P_{5}< & x_{2}-x_{1}+1+x_{4}-x_{2}+.03+\left(1+d_{3}^{2}\right)^{1 / 2} \\
& +x_{5}-x_{3}+.07 \\
& <x_{5}-x_{1}+d_{3}+\left(1+d_{3}^{2}\right)^{1 / 2}+1.1 \\
& <x_{5}-x_{1}+2.8
\end{aligned}
$$

if $d_{3} \leqq .55$. We may therefore suppose that $d_{3}>.55$. Then if $\phi$ is the 
inclination of $P_{3} P_{4}$ to the $x$-axis,

$$
\tan \phi<\frac{1}{.55}, \quad \tan \frac{\phi}{2}<.6, \quad P_{3} P_{4}<d_{3}+.6,
$$

and, by (11), $P_{1} P_{2} P_{3} P_{4}$ is a path as required.

Case $\alpha \delta \alpha \delta \beta$. Clearly,

$$
P_{1} P_{2} P_{4} P_{5} P_{3}<x_{3}-x_{1}+2\left(x_{5}-x_{3}\right)+2<x_{3}-x_{1}+2.8
$$

if $x_{5}-x_{3} \leqq .4$. We may therefore suppose that $x_{5}-x_{3}>.4$. Then if $\psi$ is the inclination of $P_{3} P_{5}$ to the $x$-axis,

$$
\tan \psi<\frac{5}{4}, \quad \tan \frac{\psi}{2}<\frac{1}{2}
$$

and

$$
P_{3} P_{6} \leqq x_{5}-x_{3}+\left(1-y_{5}\right) / 2 \leqq x_{5}-x_{3}+.25
$$

Now

$$
P_{3} P_{5} P_{4} \leqq x_{5}-x_{3}+\left(1-y_{5}\right) / 2+\left(d_{4}^{2}+y_{5}^{2}\right)^{1 / 2} \leqq d_{3}+1.3
$$

if

$$
d_{4}+\left(1-y_{6}\right) / 2+\left(d_{4}^{2}+y_{5}^{2}\right)^{1 / 2} \leqq 1.3
$$

If (12) is satisfied, then, by (11), $P_{1} P_{2} P_{3} P_{5} P_{4}$ is a path as required. But (12) is satisfied if $d_{4} \leqq 1 / 3$. It suffices to prove this when $d_{4}=1 / 3$. For this value of $d_{4}$, the first member of (12) is an increasing function of $y_{5}$ in the range of $y_{5}$, namely, $1 / 2 \leqq y_{5} \leqq 4 / 5$. It therefore suffices to prove (12) when $d_{4}=1 / 3, y_{5}=4 / 5$, in which case (12) becomes an equality.

We may therefore suppose that $d_{4}>1 / 3$. If $\omega$ is the inclination of $P_{4} P_{5}$ to the $x$-axis, then

$$
\tan \omega \leqq \frac{y_{5}}{d_{4}}<\frac{12}{5}, \quad \tan \frac{\omega}{2}<\frac{2}{3},
$$

and so

$$
P_{4} P_{5}<d_{4}+2\left(y_{5}-y_{4}\right) / 3<d_{4}+.6
$$

Now

$$
\begin{aligned}
P_{1} P_{2} P_{4} P_{3} P_{5}< & x_{2}-x_{1}+1+x_{4}-x_{2}+.03+\left(1+d_{3}^{2}\right)^{1 / 2} \\
& +x_{5}-x_{3}+.25 \\
< & x_{6}-x_{1}+2.8
\end{aligned}
$$


if $d_{3} \leqq .43$. We may therefore suppose that $d_{3}>.43$. Then

$$
\tan \phi<\frac{1}{.43}, \quad \tan \frac{\phi}{2}<.66, \quad P_{3} P_{4}<d_{3}+.66,
$$

and, by (11) and (13), $P_{1} P_{2} P_{8} P_{4} P_{6}$ is a path as required.

Case $\alpha \delta \alpha \delta \gamma$. As in the preceding case, we may suppose that $x_{5}-x_{3}>$.4. Then

$$
\begin{gathered}
\tan \psi<2, \quad \tan \frac{\psi}{2}<.62, \\
P_{3} P_{5} \leqq x_{5}-x_{3}+.62\left(y_{3}-y_{5}\right)<x_{5}-x_{3}+.5 .
\end{gathered}
$$

Now

$$
\begin{aligned}
P_{1} P_{2} P_{4} P_{3} P_{5}< & x_{2}-x_{1}+1+x_{4}-x_{2}+.03+\left(1+d_{3}^{2}\right)^{1 / 2} \\
& +x_{5}-x_{3}+.5 \\
< & x_{5}-x_{1}+2.8
\end{aligned}
$$

if $d_{3} \leqq .23$. We may therefore suppose that $d_{3}>$.23. Then

$$
\tan \phi<\frac{1}{.23}, \quad \tan \frac{\phi}{2}<.8, \quad P_{3} P_{4}<d_{3}+.8 .
$$

Further,

$$
P_{4} P_{5}<d_{4}+.5 \text {. }
$$

By (11), $P_{1} P_{2} P_{3} P_{4} P_{5}$ is a path as required.

Case $\alpha \delta \alpha \delta \delta$. We have

$$
P_{8} P_{4} P_{5}<1+d_{3}+d_{4}+.07 \text {, }
$$

so that, by (11), $P_{1} P_{2} P_{3} P_{4} P_{5}$ is a path as required.

QUEEN'S UNIVERSITY, BELFAST 